Ann. Zootech., 1988, 37 (1), 31-42

\title{
Modifications d'ingestibilité entraînées par la fenaison chez les bovins. Comparaison avec les ovins
}

\author{
J.P. DULPHY, J. ROUEL * \\ Avec la collaboration technique de J. BONY et J.M. GIRAUD \\ INRA, Laboratoire des Aliments \\ Centre de Recherches de Clermont-Ferrand-Theix, F 63122 Ceyrat \\ * INRA, Domaine d'Orcival \\ Centre de Recherches de Clermont-Ferrand-Theix, F 63210 Rochefort-Montagne
}

\begin{abstract}
Résumé
Pour connaître les modifications d'ingestibilité entrainées par la fenaison nous avons comparé les quantités ingérées de 16 foins à celles des fourrages verts à partir desquels ils avaient été préparés. Douze foins ont été récoltés dans de très bonnes conditions et 4 ont reçu de la pluie. Les fourrages verts témoins étaient fauchés chaque jour et distribués à des moutons et des génisses Holstein de 15 mois. On a considéré comme un échantillon de fourrage vert ce qui avait été fauché pendant la semaine durant laquelle le foin correspondant était préparé.
\end{abstract}

Les quantités ingérées moyennes des échantillons de fourrage vert ont été de $67 \mathrm{~g}$ de M.S. $/ \mathrm{kg} \mathrm{P} \mathrm{P}^{0.75}$ pour les moutons et $88,8 \mathrm{~g}$ pour les génisses. Pour les 12 foins préparés dans de bonnes conditions l'ingestibilité, par rapport à celle du fourrage vert, a été diminuée de 19 p. 100 chez les moutons et augmentée de 5 p. 100 chez les génisses. Pour les 4 autres foins, les diminutions ont été respectivement de 30 et de 8 p. 100.

Pour les 12 foins préparés dans de bonnes conditions les quantités ingérées par les génisses (Q.I.G.) ont été reliées à celles ingérées par les moutons (Q.I.M.) :

Q.I.M. foin $=-17,7+1,071$ Q.I.M. f. vert $\pm 5,1(\mathrm{r}=0,827)$

Q.I.G. foin $=25,6+0,766$ Q.I.M. f. vert $\pm 5,0(\mathrm{r}=0,592)$.

Compte tenu de ces résultats, il apparaît que les relations entre quantités ingérées par les ovins et les bovins doivent être calculées séparément pour les fourrages verts et les fourrages conservés.

Mots clés: Ingestion, fourrage vert, foin, génisses, moutons.

\section{Introduction}

Les principales modifications d'ingestibilité entraînées par la fenaison, et leurs causes, ont été très largement étudiées chez le mouton par Demarquilly, Andrieu et Weiss (1981). Les baisses d'ingestibilité observées varient selon la famille du végétal étudié, le mode de préparation $\mathrm{du}$ foin et les conditions climatiques lors du fanage. Aucun travail de ce type n'a encore été effectué sur bovins. Dans une étude, 
cependant, la déshydratation (VeriTe et Journet, 1970) a provoqué une augmentation faible $(+3$ p. 100) des quantités de matière sèche ingérées par les vaches laitières.

On connaît par contre ce qui se passe pour les ensilages d'herbe (Dulphy, Michalet-Doreau et Demarquilly, 1984). Chez les génisses, les ensilages préparés dans les meilleures conditions ont des ingestibilités proches de celles des fourrages verts initiaux chez les moutons; on observe toujours une diminution de l'ingestibilité des fourrages après ensilage, celle-ci pouvant avoir plusieurs causes : un rassasiement rapide lors des grands repas (Dulphy, 1985) ou une baisse de l'appétit des moutons en période de jours courts (Michalet-Doreau et Gatel, 1983). Compte tenu des réactions différentes des deux espèces animales utilisées, il s'avérait opportun d'examiner l'effet de la fenaison, cette technique n'induisant pas sur l'appétit des animaux les effets spécifiques des ensilages, dus probablement à l'ensemble des produits de fermentation qu'ils contiennent.

Enfin toute étude comparative entre ovins et bovins est importante dans la mesure où elle permet d'apporter de nouvelles précisions sur les possibilités de prévoir à partir des quantités ingérées par les ovins, les quantités ingérées par les bovins, plus difficiles à obtenir. On dispose bien sûr de quelques études concernant des bouvillons (BLAXTER, Wainman et Davidson, 1966 ; Engels et al., 1978 ; Rees et Little, 1980 ; Colovos et al., 1970 ; Siebert et KenNEdy, 1972), mais celles concernant des génisses sont pratiquement inexistantes (Dulphy et Michalet-Doreau, 1983) alors que nous venons de choisir la génisse comme animal standard dans le système révisé des unités d'encombrement (DulPhy et al., 1988).

\section{Matériel et méthodes}

Pendant 2 années de suite, en juin et en juillet, nous avons distribué à la fois à des génisses de race Pie noire et à des moutons castrés de race Texel des fourrages verts fauchés et récoltés tous les jours. Une fois par semaine une grande quantité d'herbe était fauchée pour préparer un foin. Au total on a préparé 16 foins qui ont été distribués durant l'hiver suivant l'étude du fourrage vert à des animaux, aussi semblables que possible à ceux utilisés pour les mesures en vert.

\section{A. Fourrages}

Les conditions de préparation des foins (date de coupe, durée de séchage au sol, nature du végétal et quantité de pluie reçue pendant le séchage) sont données dans le tableau 1. Ces foins ont été préparés sur les domaines INRA d'Orcival et de Laqueuille, situés à $1000 \mathrm{~m}$ d'altitude. Les échantillons de fourrage vert, fauchés chaque jour, ont été regroupés par semaine pour avoir un échantillon représentatif à la fois de ce qui était distribué aux animaux et de ce qui devait être transformé en foin. Les fourrages verts et les foins ont toujours été distribués à volonté (10 p. 100 de refus).

\section{B. Animaux}

En été, comme en hiver, nous avons utilisé des lots de 6 moutons, âgés de 3 ans, pesant en moyenne $67 \mathrm{~kg}( \pm 3)$ et placés en cage à métabolisme. Pour les fourrages 


\section{Tableau 1}

Conditions de préparation des foins étudiés.

Characteristics of studied hays.

\begin{tabular}{|c|c|c|c|c|}
\hline $\begin{array}{l}\text { Lieu et numéro } \\
\text { Site of harvest } \\
\text { and number }\end{array}$ & $\begin{array}{l}\text { Date de fauche } \\
\text { Date of harvest }\end{array}$ & $\begin{array}{c}\text { Durée de séchage } \\
\text { au sol (jours) } \\
\text { Length of drying } \\
\text { (days) }\end{array}$ & $\begin{array}{l}\text { Nature du végétal } \\
\text { Type of herbage }\end{array}$ & $\begin{array}{l}\text { Quantité de pluie } \\
\text { reçue (mm) } \\
\text { Quantity of rain } \\
\text { (mm) }\end{array}$ \\
\hline \multicolumn{5}{|l|}{ Orcival 1985} \\
\hline $\mathrm{O} 11 \ldots \ldots$ & $14-06$ & 14 & PN1 * & 95 \\
\hline $\mathrm{O} 12$ & $17-06$ & 11 & PN1 & 79 \\
\hline $\mathrm{O} 13 \ldots \ldots \ldots$ & $25-06$ & 4 & PN1 & 3 \\
\hline $014 \ldots \ldots \ldots$ & $8-07$ & 2 & PN1 & 0 \\
\hline $015 \ldots \ldots \ldots$ & $22-07$ & 1 & $\mathrm{D} 2$ & 0 \\
\hline O16 ....... & $12-08$ & 2 & PN2 & 0 \\
\hline \multicolumn{5}{|l|}{ Orcival 1986} \\
\hline $\mathrm{O} 21 \ldots \ldots \ldots$ & $23-06$ & 2 & PN1 & 0 \\
\hline $\mathrm{O} 22 \ldots \ldots \ldots$ & $28-06$ & 4 & PN1 & 0 \\
\hline $\mathrm{O} 23 \ldots \ldots \ldots$ & $5-07$ & 5 & PN1 & 10 \\
\hline $\mathrm{O} 24 \ldots \ldots \ldots$ & $8-07$ & 3 & PN1 & 0 \\
\hline $\mathrm{O} 25 \ldots \ldots \ldots$ & $19-07$ & 2 & $\mathrm{D} 2$ & 0 \\
\hline \multicolumn{5}{|l|}{ Laqueuille 1986} \\
\hline $\mathbf{L 1}_{1} \ldots \ldots$ & $23-06$ & 2 & Fl1 & 0 \\
\hline $\mathrm{L} 2 \ldots \ldots \ldots$ & $30-06$ & 2 & Fll & 24 \\
\hline L3 $\ldots \ldots \ldots$ & $5-07$ & 5 & Fl1 & 16 \\
\hline L4 $\ldots \ldots \ldots$ & $9-07$ & 2 & Fl1 & 0 \\
\hline L5 $\ldots \ldots \ldots$ & $14-07$ & 2 & Fl1 & 0 \\
\hline \multicolumn{5}{|c|}{$\begin{aligned}{ }^{*} \mathrm{PN} 1 & \text { : Prairie naturelle } 1^{\text {er }} \text { cyclc } \\
& \text { Natural grassland at first growth } \\
\mathrm{PN} 2: & \text { Prairie naturelle } 2^{\mathrm{c}} \text { cyclc } \\
& \text { Natural grassland at second growth }\end{aligned}$} \\
\hline
\end{tabular}

verts un seul lot a été utilisé pour chaque lieu. Pour les foins il y avait 2 lots en 1985 et 3 en 1986, tous les foins ayant été distribués à des animaux situés à Orcival. Dans ce cas on a vérifié, au milieu et à la fin de l'hiver, que la capacité d'ingestion des moutons était comparable entre lots et constante tout au long de l'hiver.

Les génisses, âgées de 15 à 18 mois et pesant en moyenne $398 \mathrm{~kg}( \pm 19)$ étaient 10 par lot en été, le même lot étant utilisé tout l'été dans un lieu donné. Les animaux étaient en stabulation entravée sans paille. Chaque hiver, on a utilisé deux lots de 6 génisses seulement, de même âge et de même poids (en moyenne $371 \pm 18 \mathrm{~kg}$ ) que celles utilisées en été. Chaque hiver, on a distribué un foin témoin au début, au milieu et à la fin de l'hiver pour mesurer une éventuelle évolution de la capacité d'ingestion des animaux. 


\section{Mesures}

Pour tous les animaux, nous avons mesuré les quantités de fourrage offertes et refusées. Pour les fourrages verts, les animaux ont subi une mise en régime d'une dizaine de jours en début de saison, puis les mesures ont été faites chaque jour, donc en continu, chaque période de 5 jours étant séparée de la suivante par le samedi et le dimanche.

Chaque foin a été distribué successivement pendant 3 semaines aux moutons ( 15 jours de mise en régime, 6 jours de mesure) et 4 semaines aux génisses ( 2 semaines de mise en régime et 2 semaines de mesure). La digestibilité des fourrages a été mesurée sur les moutons par collecte totale des fèces durant les 6 jours de période expérimentale.

Les quantités ingérées par les génisses ont été corrigées pour tenir compte d'une part des différences de capacité d'ingestion (en $\mathrm{g} / \mathrm{kg} \mathrm{P}^{0.75}$ ) enregistrés entre lots lors d'un même hiver, d'autre part de l'augmentation de cette capacité au cours de l'hiver, appréciée à travers les quantités de foin témoin ingérées $\left(+16 \mathrm{~g}\right.$ de M.S. $/ \mathrm{kg} \mathrm{P}^{0,75}$ en 5 mois).

Tous les animaux ont été pesés en milieu de semaine, lorsqu'il y avait des mesures de quantités ingérées.

\section{Analyses}

Sur les échantillons finaux, correspondant à une semaine de contrôle des quantités ingérées et obtenus après un séchage à $80^{\circ} \mathrm{C}$ pendant $48 \mathrm{~h}$ et broyage à la grille de $1 \mathrm{~mm}$, nous avons mesuré les teneurs en cendres, après incinération à $550^{\circ} \mathrm{C}$, en azote total par la méthode Kjeldahl, pour avoir les matières azotées totales (M.A.T. $=\mathrm{N} \times 6,25$ ) et la cellulose brute (C.B.) par la méthode de Weende.

\section{Résultats}

\section{A. Composition et valeur nutritive des fourrages}

Les caractéristiques moyennes des 16 fourrages verts témoins sont données dans le tableau 2, avec celles des foins correspondants. La fenaison a provoqué une diminution notable de la teneur en matières azotées et une augmentation de celle en cellulose brute (respectivement -8 p. 100 et +12 p. 100).

Les 4 foins ayant été affectés par la pluie (O11, O12, L2 et L3) se distinguent des autres par une augmentation beaucoup plus importante de leur teneur en cellulose brute : $+61 \mathrm{~g}$ contre $+22 \mathrm{~g}$ et une baisse un peu plus nette de leur teneur en M.A.T. : - 11,8 g contre - 10,6 g.

Ces modifications dues à la fenaison ont entraîné une baisse de la digestibilité de la matière organique, égale à 7 points pour les 4 foins affectés par la pluie et 2,7 points seulement pour les autres. Les modifications parallèles des valeurs en U.F.L./kg de M.S. ont été de $-0,067$ U.F.L./kg de M.S. ( $-0,125$ avec pluie contre 0,048 sans pluie). 


\section{TABleau 2}

Caractéristiques chimiques moyennes des fourrages verts et des foins. Mean chemical characteristics of fresh forages and hays.

\begin{tabular}{|c|c|c|c|}
\hline & $\begin{array}{c}\text { Fourrages } \\
\text { verts } \\
\text { Fresh } \\
\text { forages }\end{array}$ & $\begin{array}{l}\text { Foins } \\
\text { Hays }\end{array}$ & $\begin{array}{l}\text { Evolution } \\
\text { due à la } \\
\text { fenaison } \\
\text { Difference }\end{array}$ \\
\hline $\begin{array}{l}\text { Teneur en matière sèche }(\mathrm{g} / \mathrm{kg} \text { brut }) \ldots \\
\text { Dry matter level }(g / \mathrm{kg})\end{array}$ & $209 \pm 33$ & $854 \pm 9$ & 一 \\
\hline $\begin{array}{l}\text { Dry matter level }(\mathrm{g} / \mathrm{kg}) \\
\text { Teneur en cendres }(\mathrm{g} / \mathrm{kg} \text { M.S. }) \ldots \ldots \ldots \ldots \ldots \ldots \\
\text { Ashes }(\mathrm{g} / \mathrm{kg} \text { D.M. } \ldots \ldots \ldots\end{array}$ & $82 \pm 15$ & $81 \pm 19$ & -1.7 \\
\hline $\begin{array}{l}\text { Teneur en matières azotées totales }(\mathrm{g} / \mathrm{kg} \text { M.S. }) \ldots \ldots \\
\text { Crude protein }(\mathrm{g} / \mathrm{kg} \text { D.M. })\end{array}$ & $139 \pm 31$ & $128 \pm 28$ & \\
\hline $\begin{array}{l}\text { Teneur en cellulose brute (g/kg M.S.) } \ldots \ldots \ldots \ldots \\
\text { Crude fibre }(g / k g \quad D . M .)\end{array}$ & $274 \pm 29$ & $306 \pm 36$ & +31.7 \\
\hline $\begin{array}{l}\text { Digestibilité de la matière organique }(p .100) \ldots \ldots \\
\text { Organic matter digestibility }(p .100)\end{array}$ & $65.2 \pm 5.8$ & $61.4 \pm 4.5$ & -3.8 \\
\hline
\end{tabular}

\section{B. Quantités ingérées}

\section{Fourrages verts}

Les quantités ingérées moyennes de fourrage vert ont été significativement plus élevées pour les génisses que les moutons (tabl. 3) en g de M.S./kg P0.75. Les quantités

\section{Tableau 3}

Quantités moyennes de fourrage ingérées.

Mean forage intake.

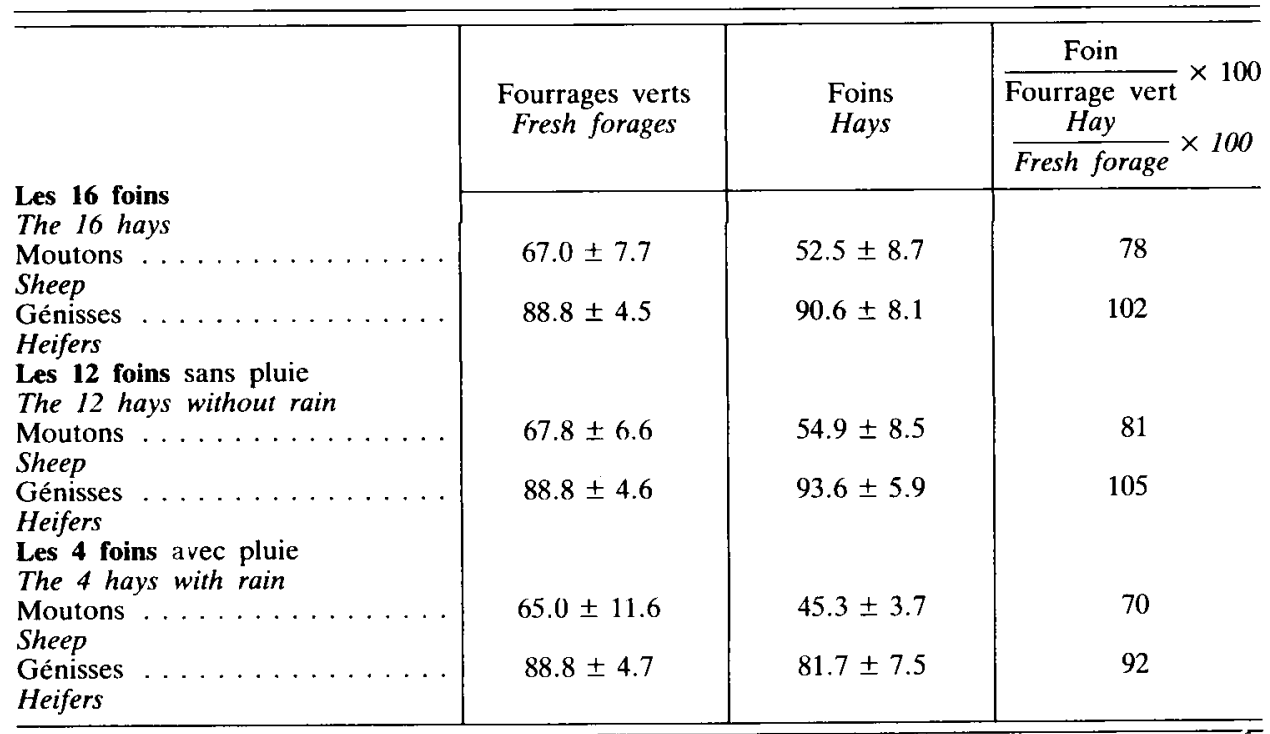


ingérées par les génisses ont peu varié avec le stade de récolte, ce qui est confirmé par le faible écart-type inter-fourrages de la moyenne.

La liaison entre les ingestibilités mesurées chez les génisses et les moutons a été la suivante :

Q.I.G. f. vert $=66,7+0,33$ Q.I.M. f. vert

$$
\pm 3,8 \quad \mathbf{r}=0,573 \quad \mathbf{n}=16
$$

Cette relation confirme que les quantités ingérées par les génisses ont été beaucoup moins variables d'une semaine à l'autre que celles des moutons.

\section{Foins}

Les foins ont également été ingérés significativement plus par les génisses que par les moutons (tabl. 3). La liaison entre les ingestibilités chez les génisses et les moutons a été la suivante (fig. 1) :

$$
\begin{aligned}
& \text { Q.I.G. foin }=64,2+0,503 \text { Q.I.M. foin } \\
& \pm 7,1 \quad \mathbf{r}=0,538 \quad \mathbf{n}=16
\end{aligned}
$$

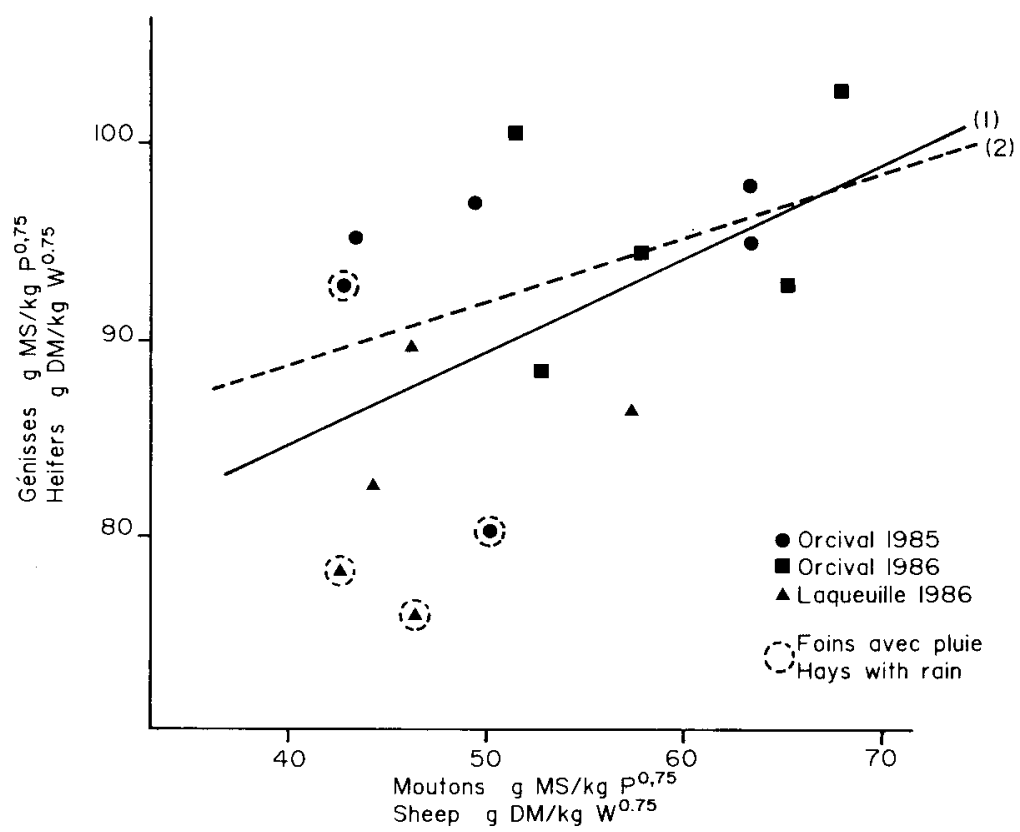

FIG. 1

Relation entre les quantités ingérées de foin par les moutons et les génisses.

Relationship between hay intake by sheep and heifers.

(1) Les 16 foins / The 16 hays.

(2) Les 12 foins préparés dans de bonnes conditions / The 12 well-cured hays. 
Cette liaison est assez lâche et la pente faible de la régression montre que l'ingestibilité des foins pour les génisses a beaucoup moins varié que celle pour les moutons. Ceci est encore plus net si on fait abstraction des 4 foins (O11, O12, L2 et L3) qui ont été nettement affectés par la pluie (fig. 1) :

$$
\begin{array}{ccc}
\text { Q.I.G. foin }=76,2+ & 0,316 \text { Q.I.M. foin } & \\
& \pm 5,5 & \mathbf{r}=0,455
\end{array} \quad \mathrm{n}=12
$$

\section{Effet de la fenaison}

Pour les 12 foins préparés dans de bonnes conditions (sans pluie) l'effet de la fenaison sur l'ingestibilité des fourrages est très variable selon l'espèce animale (tabl. 3) : diminution de 19 p. 100 chez les moutons et augmentation de 5 p. 100 chez les génisses.

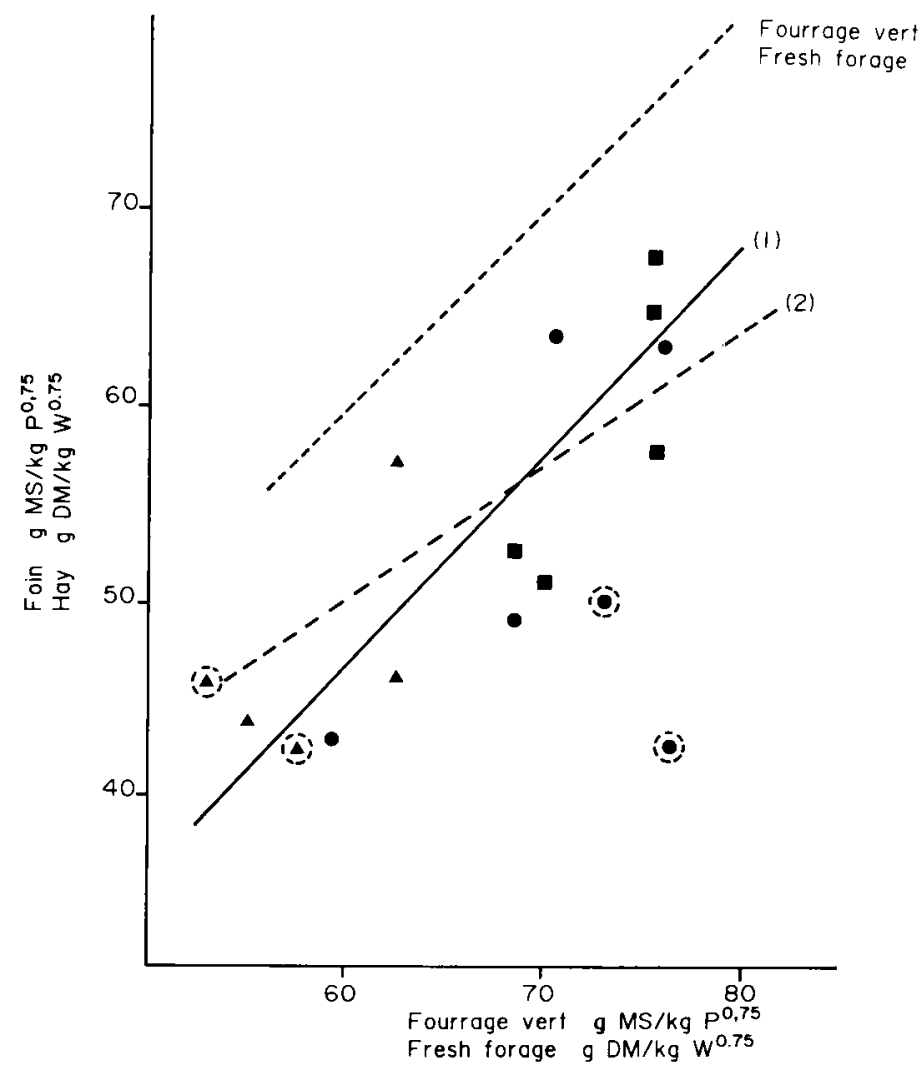

FIG. 2

Influence de la fenaison sur les quantités ingérées chez le mouton.

Influence of haymaking on D.M. intake in sheep.

(1) Foins bien préparés dans cet essai / Well-cured hays in this trial.

(2) Relation de Demarquilly et al. (1984) / Well-cured hays of Demarquhly et al. (1984). 
Les relations entre les quantités ingérées de fourrage vert et de foin sont alors les suivantes (fig. 2 et 3 ) :

$$
\begin{aligned}
& \text { Q.I.M. foin }=-17,7+1,071 \text { Q.I.M. f. vert } \pm 5,1 \\
& \mathbf{r}=0,827 \\
& \mathrm{n}=12 \\
& \text { Q.I.G. foin }=25,6+0,766 \text { Q.I.G. f. vert } \pm 5,0 \\
& r=0,592 \\
& \mathrm{n}=12
\end{aligned}
$$

Pour les fourrages qui ont reçu la pluie $(n=4)$ on observe une diminution d'ingestibilité de 30 p. 100 chez les moutons et 8 p. 100 chez les génisses (11 p. 100 pour les 3 foins les plus affectés).

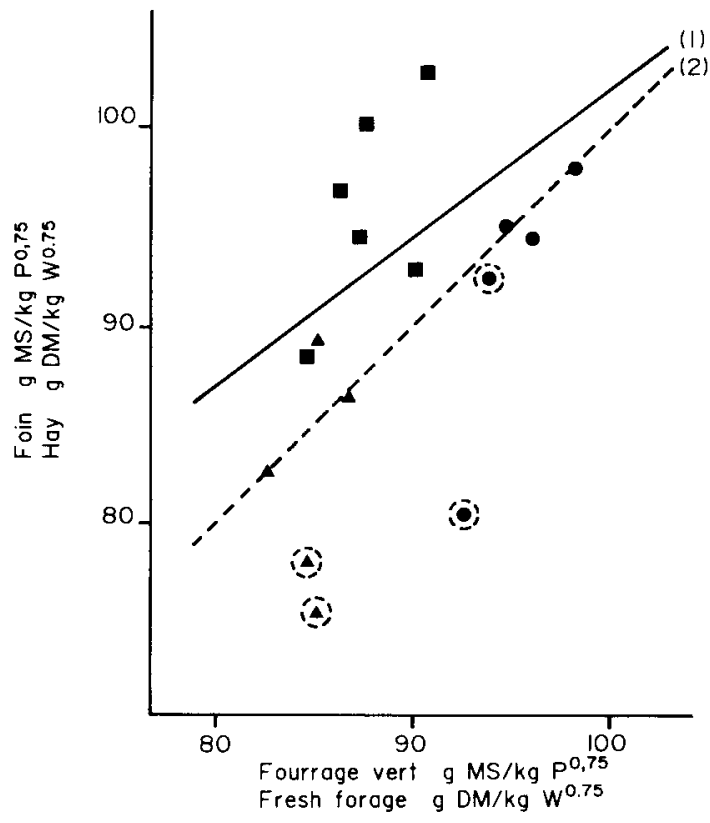

FIG. 3

Influence de la fenaison sur les quantités ingérées chez la génisse.

Influence of hay-making on D.M. intake in heifers.

(1) Foins bien préparés / Well-cured hays.

(2) Fourrage vert / Fresh forage.

\section{Discussion - Conclusion}

Compte tenu du faible nombre de foins étudiés (16) on pourrait s'interroger sur la validité des résultats. Dans leur étude réalisée sur des moutons, Demarquilly, Andrieu et Weiss (1981) ont trouvé que l'ingestibilité (en matière sèche) du foin séché au sol (Q.I. foin) était reliée à celle du fourrage vert (Q.I. f. vert) par la relation :

Q.I. foin $=8,5+0,69$ Q.I. f. vert 
On avait alors en moyenne la quantité de foin ingérée égale à 81 p. 100 de celle du fourrage vert.

Les résultats que nous obtenons sur les moutons sont semblables. En conséquence, nous pouvons considérer que les 12 foins préparés dans de bonnes conditions sont représentatifs. Dans ce cas on peut admettre que la fenaison ne modifie pas l'ingestibilité des fourrages chez les génisses, voire l'augmente. Il est probable qu'il en est de même pour tous les bovins, comme le trouvent VeriTe et Journet (1970) avec des fourrages déshydratés, proches de nos bons foins, distribués à des vaches laitières.

Ces observations entraînent deux commentaires. Le premier est la différence de réaction très importante entre moutons et génisses. Le second est que ce qui se passe pour le foin chez les génisses est proche de ce qui se passe pour les ensilages. Nous avons trouvé en effet que les ensilages d'herbe préparés dans de bonnes conditions (Dulphy, Michalet-Doreau et Demarquilly, 1984) étaient ingérés de façon comparable aux fourrages verts initiaux.

En ce qui concerne le premier point, il est à peu près certain que nous nous trouvons confrontés à un effet saison auquel les moutons sont très sensibles, mais pas les génisses. En effet, les moutons que nous utilisons ingèrent plus un même fourrage en jours longs qu'en jours courts (Michalet-Doreau et Gatel, 1983). On peut en tirer une conséquence générale : la prévision des quantités ingérées par les bovins à partir des ovins doit être effectuée séparément pour les fourrages verts, pour les foins et pour les ensilages.

En ce qui concerne le second point, il se confirme qu'il y a similitude, chez les bovins, entre les quantités ingérées de fourrage vert et de fourrage conservé, à condition que ce dernier soit préparé dans d'excellentes conditions. Dans ce cas, les facteurs régulant l'ingestion sont, avant tout, d'ordre digestif et il est probable que les vitesses de digestion sont alors très proches pour les fourrages verts et les fourrages conservés correspondants bien préparés.

Dans notre étude, les foins ont tendance à être mieux ingérés que les fourrages verts. On peut l'attribuer à deux causes. Dans certains cas la quantité d'eau contenue dans la plante peut être un facteur d'encombrement qui limite l'ingestion (VerIrE et Journet, 1970). C'est ce que traduit, de façon indirecte (Dulphy et D'Hour, 1987) la pente de 1,62 entre la teneur en matière sèche (en p. 100) et l'ingestibilité de l'herbe chez les génisses. Ceci donne une possibilité d'écart de 10 p. 100 de quantités de matière sèche ingérées pour le même fourrage lorsque la teneur en matière sèche varie de 5,5 points aux alentours de $18 \mathrm{p}$. 100. La seconde cause pourrait être que les animaux ont plus de temps en hiver pour s'adapter aux fourrages étudiés. A cause de cela ou par suite d'une adaptation aux conditions de conduite hivernale on observe d'ailleurs que la capacité d'ingestion rapportée au point métabolique, augmente d'environ 10 p. 100 entre le début et la fin de l'hiver. Des incertitudes pèsent donc sur les comparaisons de mesures de quantités ingérées faites, les unes en été et les autres en hiver, chacune restant cependant valable dans son contexte.

Dans notre étude préalable effectuée avec des ensilages d'herbe (DuLPhy, MichaLET-Doreau et Demarquilly, 1984) les génisses avaient ingéré sous forme d'ensilage à brins courts et très bien conservés $98 \mathrm{p} .100$ de la matière sèche ingérée en vert. Dans l'étude présente, le pourcentage pour les foins est de $105 \mathrm{p}$. 100 . Les bons foins auraient donc tendance à être ingérés en plus grande quantité que les bons ensilages, ce qui rejoint les résultats déjà connus de comparaisons directes (AGABRIEL, DulphY et Micol, 1987). 
En ce qui concerne les foins récoltés dans de mauvaises conditions, leur niveau d'ingestion a été inférieur de $14 \mathrm{p}$. 100 chez les moutons et de $12 \mathrm{p} .100 \mathrm{chez}$ les génisses à celui des bons foins. Demarquilly, Andrieu et Weiss (1981) ont rapporté une diminution de même amplitude. La diminution a donc été comparable pour les deux catégories d'animaux. Elle résulte probablement d'une baisse de la vitesse de digestion et des qualités organoleptiques des fourrages.

En définitive, il se confirme que, pour prévoir les quantités ingérées par des bovins, l'utilisation de mesures faites sur les moutons est possible, mais dans des conditions bien précises, c'est-à-dire, à partir de fourrages bien définis et distribués la même saison.

Reçu en octobre 1987.

Accepté en janvier 1988.

\section{Summary}

\section{Effect of wilting on changes in the voluntary feed intake in cattle as compared to sheep}

With the aim of determining the modifications of voluntary forage intake induced by wilting, the level of intake of 16 hays was compared to that of fresh forage from which they had been prepared. Twelve hays were harvested in very good conditions and 4 during rain. The fresh control forages were harvested every day and offered to sheep and 15-month old Holstein heifers. The fresh forage sample was cut during the same week as the corresponding hay was prepared.

The mean amounts ingested of fresh forage were $67 \mathrm{~g} \mathrm{D.M.} / \mathrm{kg} \mathrm{W}^{0.75}$ for sheep and $88.8 \mathrm{~g}$ for heifers. As regards the 12 hays prepared in good conditions, the voluntary intake relative to that of fresh forage was reduced by 19 p. 100 in sheep and by 5 p. 100 in heifers. The reductions were 30 and $8 \mathrm{p}$. 100 , respectively for the other 4 hays.

Concerning the 12 hays prepared in good conditions the amounts ingested by heifers (Q.I.G.) were related to those ingested by sheep (Q.I.M.) :

Q.I.M. hay $=-17.7+1.071$ Q.I.M. fresh f. $\pm 5.1(\mathrm{r}=0.827)$

Q.I.G. hay $=25.6+0.766$ Q.I.M. fresh f. $\pm 5.0(\mathrm{r}=0.592)$.

These results show that amounts ingested by sheep and cattle should be calculated separately for fresh and stored forages.

Key words: Ingestion, green forage, hay, heifers, sheep.

\section{Références bibliographiques}

Agabriel J., Dulphy J.P., Micol D., 1987. Utilisation des foins pour la croissance et l'engraissement des bovins. In $\mathrm{C}$. Demarquilly $\mathrm{Ed}$., Les fourrages secs : récolte, traitement, utilisation. INRA Paris, 283-318.

Blaxter K.L., Wainman F.W., Davidson J.L., 1966. The voluntary intake of food by sheep and cattle and relation to their energy requirements for maintenance. Anim. Prod., 8, 75-83.

Colovos N.F., Holter J.B., Koes R.M., Urban Jr W.E., Davis H.A., 1970. Digestibility, nutritive value and intake of ensiled corn plant (Zea Mays) in cattle and sheep. J. Anim. Sci., 30, $819-824$. 
Demarquilly C., Andrieu J., Weiss Ph., 1981. L'ingestibilité des fourrages verts et des foins et sa prévision. In Prévision de la valeur nutritive des aliments des ruminants. Ed. INRA Publications, Route de St-Cyr, 78000 Versailles, 155-168.

Dulphy J.P., 1985. Etude des quantités ingérées lors des grands repas chez des moutons recevant des fourrages ensilés. Ann. Zootech., 34, 401-416.

Dulphy J.P., D'Hour P., 1987. Comparaison des quantités de fourrage vert ingérées entre des vaches laitières, des génisses de race laitière, des génisses de race à viande et des moutons. Reprod. Nutr. Dévelop., 27, 209-210.

Dulphy J.P., Faverdin Ph., Micol D., Bocquier F., 1988. Révision du système des unités d'encombrement. Bull. Tech. C.R.Z.V. Theix, INRA, 70, 35-48.

Dulphy J.P., Michalet-Doreau B., 1983. Comportement alimentaire et mérycique d'ovins et de bovins recevant des fourrages verts. Ann. Zootech., 32, 465-474.

Dulphy J.P., Michalet-Doreau B., Demarquilly C., 1984. Etude comparée des quantités ingérées et du comportement alimentaire et mérycique d'ovins et de bovins recevant des ensilages d'herbe réalisés selon différentes techniques. Ann. Zootech., 33, 291-320.

Engels E.A.N., Ferreira B., Swart J.A., Nieman P.J., 1978. Comparative feed intake and digestibility studies with sheep and cattle on roughages. S. Afr. J. Anim. Sci., 8, 149-152.

Michalet-Doreau B., Gatel F., 1983. Evolution au cours d'une année des quantités de foin ingérées par des béliers castrés. Ann. Zootech., 32, 459-464.

ReEs M.C., LitTle D.A., 1980. Differences between sheep and cattle in digestibility, voluntary intake and retention time in the rumen of three tropical grasses. J. Agric. Sci., 94, 483-485.

Siebert B.D., Kennedy P.M., 1972. The utilization of spear grass (Heteropogon contortus). I. Factors limiting intake and utilization by cattle and sheep. Aust. J. Agric. Res., 23, 35-44.

Verite R., Journet M., 1970. Influence de la teneur en eau et de la déshydratation de l'herbe sur sa valeur alimentaire pour les vaches laitières. Ann. Zootech., 19, 255-268. 